missing data is found to be at random. Data from external sources like UNAIDS, World Bank and IMF will also be used for comparison and validation of TESSy data for imputation of missing data. Continuous variables will be analyzed through appropriate parametric and non-parametric tests while categorical variables will be analyzed through methods of proportion. Multivariate logistic regression methods will be used to explore the associations between VL testing and VL suppression separately with age, sex, year of diagnosis, country of origin (migrants), mode of transmission, in the total population, then at country- and regional-level. The same associations will be explored using a country's EU and EEA status (EU versus EEA versus non-EU/EEA), and income status (high versus upper middle versus lower middle versus low). DISCUSSION/ SIGNIFICANCE OF IMPACT: Even though this is a retrospective analysis of a database with likely significant missing data that may affect analysis of data and interpretation of results, our study will impact all levels of HIV policy across Europe. The strengths of this study likely outweigh the limitation imposed by missing data and include potential regional-, country- and demographic-specific public health, epidemiologic and ART program policy initiatives. Also our analysis of pattern of missing data may inform a more efficient and meaningful data collection and input into TESSy database.

\section{U.S. Counties with High Opioid-Overdose Mortality and Low Capacity to Deliver Medications for Opioid Use Disorder: an Observational Study \\ Rebecca Haffajee ${ }^{1}$, Lewei Allison Lin, M.D., M.S, Amy S.B. Bohnert, Ph.D., M.H.S. and Jason E. Goldstick, Ph.D. ${ }^{1}$ University of Michigan School of Medicine}

OBJECTIVES/SPECIFIC AIMS: To identify characteristics of counties with persistently high opioid-overdose rates and low capacity to deliver medications for OUD (MOUD). METHODS/ STUDY POPULATION: Setting: County-level opioid-overdose death data, 2013-2016, and 2017 publicly-available treatment provider data for MOUD: buprenorphine-waivered providers, opioid treatment programs (OTPs), and extended-release naltrexone providers. Participants: Populations in 3,142 U.S. counties. 24,851 buprenorphine-waivered providers; 1,517 OTPs; and 5,222 extendedrelease naltrexone providers. Measurements: The outcome variable, "opioid high-risk county", was a binary indicator of high (above average) opioid-overdose rates with low (below median) MOUD availability rates. We used spatial logistic regression models to determine correlates of being a high-risk county. RESULTS/ ANTICIPATED RESULTS: $46.4 \%$ of all counties, and $71.2 \%$ of rural counties, lacked a publicly-available MOUD provider in 2017. In adjusted models, rural counties had $53 \%$ greater odds of being high-risk than urban counties. Counties in the East South Central, West South Central, and South Atlantic divisions had over twice the odds of being high-risk than counties in the West North Central division. Primary care provider density, greater traversability, and a higher proportion of the population under age 25 were all protective against a county being opioid high-risk. DISCUSSION/ SIGNIFICANCE OF IMPACT: Counties with both low MOUD provider availability and high opioid-overdose death rates are significantly more likely to be rural, have less primary care providers per capita, and in the southern regions. Strategies to increase MOUD must account for these factors.

\section{Team Science}

\section{A TL1 Team Approach to Clinician Perspectives on Hoarding Disorder \\ Rebecca R Henderson ${ }^{1}$, Danielle Laine Cooke ${ }^{1}$, Christian Archer $^{1}$, Joseph P. McNamara ${ }^{1}$, Pete Collings ${ }^{1}$ and Carol Mathews ${ }^{1}$ \\ ${ }^{1}$ University Of Florida}

OBJECTIVES/SPECIFIC AIMS: By combining clinical knowledge of hoarding disorder (HD) with qualitative methods from cultural anthropology, we hope to build a patient-centered approach that will allow us to better understand the clinician perspectives on patient motivations and explanatory models of individuals with HD, and improve treatment outcomes. We describe the ways that these methodologies are productively merged in this project as a result of TL1 collaboration, and present a preliminary picture of methodological and theoretical issues uncovered as part of this processes. We further describe the analytical methods used for this project, and explore issues raised through the combination of psychological and anthropological data and insights. METHODS/STUDY POPULATION: This study represents an attempt to combine the qualitative methodologies of cultural anthropology with the clinical knowledge of psychology and psychiatry in order to better understand gaps between provider and patient beliefs and knowledge about hoarding disorder. This study will present preliminary methodological issues arising from interviews with hoarding experts. RESULTS/ANTICIPATED RESULTS: This study will discuss preliminary issues including shared language, strengths and limitations of both disciplines, and factors for consideration when combining these disparate methodologies. It will close with recommendations for consideration when moving forward with similar collaborations. DISCUSSION/ SIGNIFICANCE OF IMPACT: This project seeks to unite psychological and social factors that may contribute to the lived experience of individuals with HD in order to better understand the way that $\mathrm{HD}$ is manifested. It also unites disparate methodologies to provide us with a more holistic and complete picture of the experience of HD. While HD has been studied within psychiatry, it has never been assessed using the qualitative methods of anthropology. These methods provide the possibility of expanding knowledge about the ways that this disorder is experienced by individuals and their families, and potentially impacted by shared beliefs and cultures. Furthermore, qualitative data of this nature provides a patient perspective on the experience of HD as a psychiatric illness. This patient perspective can be used to better inform treatment, improve patient outcomes, and to allow providers and researchers to gain a fuller understanding of this complex population.

3475

\section{A TL1 Team Approach to CNS-Localized Delivery of Neurotrophic Factors for Treatment of Parkinson's Disease \\ Adithya Gopinath ${ }^{1}$ and Shaheen Farhadi \\ ${ }^{1}$ University Of Florida Clinical and Translational Science Institute}

OBJECTIVES/SPECIFIC AIMS: We present an alternative strategy to retain NTFs at an injected CNS tissue site by endowing them with binding affinity for carbohydrates that are abundant on the cell surface and within extracellular matrices. METHODS/STUDY 